gr-qc/9806105

\title{
Quantization of pure gravitational plane waves
}

\author{
Guillermo A. Mena Marugán \\ I.M.A.F.F., C.S.I.C., Serrano 121, 28006 Madrid, Spain \\ Manuel Montejo \\ Universitat de Barcelona, Diagonal 647, 08028 Barcelona, Spain
}

(25 June 1998)

\begin{abstract}
Pure gravitational plane waves are considered as a special case of spacetimes with two commuting spacelike Killing vector fields. Starting with a midisuperspace that describes this kind of spacetimes, we introduce gauge-fixing and symmetry conditions that remove all non-physical degrees of freedom and ensure that the classical solutions are plane waves. In this way, we arrive at a reduced model with no constraints and whose only degrees of freedom are given by two fields. In a suitable coordinate system, the reduced Hamiltonian that generates the time evolution of this model turns out to vanish, so that all relevant information is contained in the symplectic structure. We calculate this symplectic structure and particularize our discussion to the case of linearly polarized plane waves. The reduced phase space can then be described by an infinite set of annihilation and creation like variables. We finally quantize the linearly polarized model by introducing a Fock representation for these variables.
\end{abstract}

PACS number(s): 04.60.Ds, 04.30.-w

\section{INTRODUCTION}

The analysis of gravitational waves has received lately a great deal of attention, both from the theoretical and the experimental points of view [1]. In particular, there is an increasing interest in the study and quantization of spacetimes with two commuting Killing vectors that describe wave solutions in source-free general relativity [2-5]. One of the reasons for this interest, apart from possible applications in cosmology and astrophysics, is that this kind of spacetimes provides a good arena to test quantization techniques and discuss conceptual issues in gravity. The symmetry reduction of Einstein gravity in the presence of two commuting Killing vectors leads in general to models with an infinite number of degrees of freedom, i.e., to midisuperspace models. It is commonly accepted that the quantization of these models, which is given by a true quantum field theory, may be significant for discussing basic features of the outstanding theory of quantum gravity. In contrast, minisuperspace truncations of general relativity, like, e.g., most of the gravitational systems quantized in the literature [6], are too simple to capture the field complexity that should be present in full quantum gravity. Another motivation for studying the reduction of general relativity by two commuting spacelike Killing vectors is that it admits a similar formulation to that for coset space $\sigma$-models coupled to two-dimensional gravity and a dilaton [7]. The quantization of any of these two types of systems should hence allow a better understanding of the quantum physics of the other.

The spacetimes with two commuting Killing vectors that have been quantized so far are the family of cylindrically symmetric gravitational waves and the Gowdy model with the spatial topology of a three-torus. The
Gowdy universes are vacuum spacetimes with compact sections of constant time [8]. They can be thought of as inhomogeneous spacetimes filled with gravitational waves. The quantization of the Gowdy model when the topology of the sections of constant time is that of a three-torus was analyzed in Ref. [2]. Preliminary studies of this quantization had been carried out by Berger [9] (assuming that the Killing vectors of the model were hypersurface orthogonal), and by Husain and Smolin 10. On the other hand, the quantization of cylindrical gravitational waves with linear polarization was achieved by Ashtekar and Pierri [3], completing previous works on the subject by Kuchař [ $[4$ and Allen [11]. The effects produced by quantum fluctuations in this cylindrically symmetric model were studied in Ref. [12]. Finally, the more general case of cylindrical waves with two polarizations was recently quantized by Korotkin and Samtleben 5.

In addition to possessing two commuting spacelike Killing vector fields, the above families of spacetimes satisfy another condition: the existence of two-surfaces that are everywhere orthogonal to the group orbits spanned by the Killing vectors. For spacetimes of this class, a specially useful metric function is the square root of the determinant of the two-metric that corresponds to the group orbits 13]. We will denote this positive function by $W$. The spacelike, timelike, or null character of the gradient of $W$ is invariant under coordinate transformations. In this paper, we will study the quantization of another example of gravitational waves that belongs to this class of spacetimes, namely, the case of pure gravitational plane waves $[13,14$. Our analysis will exhaust the discussion of the different posibilities for the gradient of $W$, in the sense that this gradient is timelike for the Gowdy model with the topology of a three-torus [8], 
spacelike for cylindrical gravitational waves [3], and null for plane waves.

Gravitational plane waves are a special type of $\mathrm{pp}-$ waves, i.e., plane-fronted gravitational waves with parallel rays 13,15. They were first studied by Baldwin and Jeffrey 16., and admit a five-dimensional group of isometries with a three-dimensional Abelian subgroup that acts on null hypersurfaces [17. Gravitational plane waves can be interpreted as describing the gravitational field at great distances from finite radiating bodies 13, 16, Some of these waves are related to certain limits of Bianchi cosmological models and inhomogeneous generalizations of such models 14,18. On the other hand, the collision of two gravitational plane waves has received intensive study, and a lot of solutions that represent this collision are known [14,19].

In this work, we will restrict our considerations to the case of pure gravitational plane waves, namely, plane waves that are solutions to Einstein equations in vacuum. Preliminary studies of the quantization of spacetimes with the symmetry of these waves have been carried out by Neville 20,21 and Borissov 22, mainly in the context of the Ashtekar formalism for gravity. Here, we will start with the geometrodynamic formulation for general relativity, and discuss the quantization of pure gravitational plane waves as a particular case of spacetimes that possess two commuting spacelike Killing vectors.

The rest of the paper is organized as follows. The geometrical properties of pure gravitational plane waves are reviewed in Sec. II. With a suitable choice of (local) coordinates, we cast the metric of this family of solutions in a convenient $(3+1)$ form. This metric can be written in terms of two arbitrary functions, and corresponds to spacetimes with two commuting Killing vector fields that are subject to certain conditions. In Sec. III, we consider the Hamiltonian formulation for spacetimes that admit two commuting spacelike Killing vectors and perform a partial gauge fixing. The reduction of the system is completed in Sec. IV, where we impose gauge-fixing and symmetry conditions that remove all non-physical degrees of freedom and guarantee that the classical solutions are precisely pure gravitational plane waves. We determine the reduced phase space of the model obtained in this way and its symplectic form. It is also shown that, in an appropriate set of coordinates, the dynamical evolution of this reduced model is trivial, so that all relevant information about the system is in fact contained in the symplectic structure. In Sec. V, we particularize our study to the family of linearly polarized plane waves in vacuum gravity. We prove that the symplectic form on the reduced phase space of this model can be regarded as that corresponding to an infinite set of annihilation and creation like variables. We then proceed to quantize the model by introducing a Fock representation. Finally, we summarize our results and conclude in Sec. VI.

\section{PURE GRAVITATIONAL PLANE WAVES}

Gravitational plane waves are solutions to Einstein field equations that possess a five-parameter group of isometries [17]. For these spacetimes, the metric can always be written in the form [13]:

$$
d s^{2}=-d U d V+H_{a b}(U) X^{a} X^{b} d U^{2}+\sum_{a}\left(d X^{a}\right)^{2},
$$

where we have employed the index notation $a, b=1,2$. The coordinates $\left\{U, V, X^{1}, X^{2}\right\}$ are called harmonic coordinates, and run over the whole real axis. It is easy to check that $\partial_{V}$ corresponds to a covariantly constant null vector field, so that metrics (2.1) are a special class of pp-waves 13. On the other hand, the only nonvanishing component of the Ricci tensor for these metrics is $R_{U U}=-\left(H_{11}+H_{22}\right)$. Solutions to Einstein equations in vacuum are hence given by all symmetric matrices $H_{a b}$ with vanishing trace. In this case, the plane wave is said to be purely gravitational.

Although the use of harmonic coordinates for plane waves has clear advantages, because they allow to cover the totality of the spacetime with a single chart and express the Ricci tensor in terms of a simple metric function, the spacetime symmetries become much more apparent in the so-called group coordinates. In such coordinates, the metric reads 13,14 :

$$
d s^{2}=-d U d \bar{V}+h_{a b}(U) d x^{a} d x^{b} .
$$

Here, $\bar{V}, x^{1}$, and $x^{2}$ run over the entire real line, and the coordinate $U$ has in general a restricted range, as we will see below. Obviously, $\partial_{x^{1}}$ and $\partial_{x^{2}}$ are two commuting spacelike Killing vector fields for the above metrics, and the group orbits spanned by them are everywhere orthogonal to the surface with coordinates $U$ and $\bar{V}$. It is convenient to write the two-metric $h_{a b}$ of these group orbits as

$$
\begin{aligned}
& h_{11}=e^{z-y / 2}, \quad h_{12}=-v e^{z-y / 2}, \\
& h_{22}=\left(v^{2}+e^{y}\right) e^{z-y / 2} .
\end{aligned}
$$

Hence, the condition that $h_{a b}$ is positive definite is automatically satisfied provided that the functions $v, y$, and $z$ are real. Note that the determinant of $h_{a b}$ is equal to $e^{2 z}$. Thus, from our discussion in the Introduction, we have $W=e^{z}$ and, since this function depends only on the coordinate $U$, its gradient is null with respect to the metric (2.2), as we had anticipated. Finally, the functions $v$ and $y$ describe, respectively, the diagonal and non-diagonal degrees of freedom of the metric $e^{-z} h_{a b}$, which has unit determinant.

In group coordinates, the only non-trivial Einstein equation in vacuum is

$$
R_{U U}=-\frac{1}{2} h^{a b} \frac{d^{2} h_{a b}}{d U^{2}}-\frac{1}{4} \frac{d h^{a b}}{d U} \frac{d h_{a b}}{d U}=0,
$$


with $h^{a b}$ being the inverse of the two-metric $h_{a b}$. Substituting Eq. (2.3) in this expression, we get

$$
\frac{d^{2}\left(e^{z / 2}\right)}{d U^{2}}=-\frac{e^{z / 2}}{16}\left[\left(\frac{d y}{d U}\right)^{2}+4\left(\frac{d v}{d U}\right)^{2} e^{-y}\right] .
$$

Therefore, $W^{1 / 2}=e^{z / 2}$ must be a convex function of the coordinate $U$. Since this function is non-negative, it must then generally vanish at some point. The metric becomes degenerate at that point and a coordinate singularity appears. As a consequence, the spacetime for pure gravitational plane waves cannot be described by a unique chart in group coordinates [14].

Actually, the fact that $W^{1 / 2}$ is a non-negative convex function implies that it must vanish at either one or two points in the allowed (simply connected) interval of variation for the coordinate $U$. The only exception is pure Minkowski spacetime, where $v, y$, and $z$ are constant. When $W^{1 / 2}$ has two zeros, it increases from the first of them until a maximum is reached. This maximum can always be made equal to the unity by means of a suitable rescaling of the real coordinates $x^{a}$. On the other hand, when $W^{1 / 2}$ has a single zero, it can be chosen as a strictly increasing function of $U$ by performing a reversal of the null coordinates $U$ and $V$, if necessary. In this case, $W^{1 / 2}$ will either tend to infinity or to a finite value in the limit of large positive $U$ 's. If the limit is finite, an appropriate rescaling of $x^{a}$ sets it equal to the unity. Hence, we see that in all cases there exists a region of the spacetime where the function $W^{1 / 2}$ increases with $U$ and ranges in the interval $(0,1)$. In this region, $z$ is strictly increasing from minus infinity to zero. In addition, although Minkowski spacetime has apparently been excluded from our discussion, it turns out that the above considerations apply as well to flat spacetime, because the solution to Eq. (2.5) in which $v$ and $y$ are constant and $W^{1 / 2}$ is linear in $U$ can be cast in Minkowskian form by a (local) coordinate transformation [17].

In the region where $z$ increases in $(-\infty, 0)$, we can introduce a change of coordinates

$$
d U=e^{2 w-z+y / 2} d u
$$

so that the function $z$ gets fixed in terms of our new coordinate $u$. For instance, we can make

$$
z(U[u])=-e^{-u} \equiv z_{0}(u) .
$$

In Eq. (2.6), $w$ is an unknown function of $u$. This coordinate is assumed to run over the entire real axis. Our change of coordinates is then well-defined and invertible, because $z_{0}$ is strictly increasing for $u \in \mathbb{R}$ and has the range $\mathbb{R}^{-}$. With this change, the coordinate singularity at $e^{z}=0$ has been driven to minus infinity. Notice also that our ignorance about the original function $z(U)$ is encoded in $w$. However, the vacuum Einstein equation (2.5) becomes now a first-order differential equation in $w$ that can be solved explicitly. The solution is

$$
\begin{gathered}
w=\frac{\ln \left(z_{0}^{\prime}\right)}{2}+\frac{3 z_{0}}{4}-\frac{y}{4}+\int_{u_{0}}^{u} \frac{1}{16 z_{0}^{\prime}}\left[\left(y^{\prime}\right)^{2}+4\left(v^{\prime}\right)^{2} e^{-y}\right] \\
\equiv w_{0},
\end{gathered}
$$

where $u_{0}$ is any fixed real constant and the prime stands for derivative with respect to $u$. The constant of integration that should appear in this formula has been absorbed by a scale transformation in the coordinate $\bar{V}$.

If we finally define a time via $\bar{V}=2 t-u$, we arrive at the following line element for pure gravitational plane waves:

$$
d s^{2}=e^{2 w_{0}-z_{0}+y / 2}\left[-d t^{2}+(d u-d t)^{2}\right]+h_{a b} d x^{a} d x^{b},
$$

with $h_{a b}$ the metric obtained from Eq. (2.3) by setting $z=z_{0}$. All coordinates in this expression are real and have unrestricted ranges. Recalling relations (2.7) and (2.8), we see that this family of vacuum solutions have only two degrees of freedom, which are given by the functions $v$ and $y$ of the coordinate $u$.

These are the spacetimes that we will study in the rest of this paper. As we have shown, the above metric describes the most general pure gravitational plane wave solution, with the only caveat that it does not correspond to the whole of the spacetime that can be covered with harmonic coordinates. The coordinate system that we have adopted is in fact quite similar to that employed in Ref. 17], the main difference being that we have chosen $z_{0}=-e^{-u}$ and allowed $u$ to take all real values, while $z_{0}$ was made equal to $2 \ln |u|$ in Ref. [17]. In this latter case, $u$ has a restricted range [e.g., $u \in(0,1)$ for $z_{0} \in \mathbb{R}^{-}$].

We can regard Eq. (2.9) as providing the standard $3+1$ decomposition of the metric for spacetimes with two orthogonal surfaces, but with the lapse and (non-vanishing component of) the shift vector particularized to take the values $N=e^{w_{0}-z_{0} / 2+y / 4}$ and $N^{u}=-1$. In addition, the spacetimes considered admit two commuting spacelike Killing vector fields, $\partial_{x^{1}}$ and $\partial_{x^{2}}$, and satisfy another condition, namely, that the metric functions are independent not only of $x^{a}$, but also of the time coordinate $t$. In the Hamiltonian formalism for general relativity, there is a simple way of imposing the time independence of the metrics (2.9) on classical solutions, once the existence of the two spacelike Killing vectors has been assumed. We first note that $z_{0}$ is a fixed function of $u$, and that $w_{0}$ is determined by the vacuum Einstein equations to be given by Eq. (2.8) in terms of $v$ and $y$. It hence suffices to ensure that the time derivatives $\dot{v}$ and $\dot{y}$ vanish on classical solutions. Employing then the definition of the extrinsic curvature $K_{i j}$ [23], with $x^{i} \equiv\left\{x^{1}, x^{2}, u\right\}$, it is not difficult to check that, for the metrics under discussion, the requirements $\dot{v}=\dot{y}=0$ on classical solutions are equivalent to the relations

$$
\begin{aligned}
& 4 h^{1 / 2} e^{-2 z_{0}+y / 2} K_{11}=2 z_{0}^{\prime}-y^{\prime}, \\
& 4 h^{1 / 2} e^{-2 z_{0}+y / 2} K_{12}=v y^{\prime}-2 v z_{0}^{\prime}-2 v^{\prime},
\end{aligned}
$$

where $h=e^{2 w_{0}+z_{0}+y / 2}$ is the determinant of the induced metric $h_{i j}$. Moreover, these relations turn out to imply 
the vanishing of $\dot{v}$ and $\dot{y}$ even when the values of $w_{0}$, the lapse function, and the shift component $N^{u}$ are changed in the metrics (2.9), provided that $N^{u}$ does not depend on the coordinates $x^{a}$ and the quotient $N / N^{u}=-h^{1 / 2} e^{-z_{0}}$ is not modified.

\section{SPACETIMES WITH TWO KILLING VECTOR FIELDS}

In this section, we will discuss the Hamiltonian formulation for spacetimes that possess two commuting spacelike Killing vectors, paying a special attention to the case of pure gravitational plane waves.

We will only consider spacetimes that admit a global foliation, so that the metric can be written in the $(3+1)$ form:

$$
d s^{2}=-N^{2} d t^{2}+h_{i j}\left(d x^{i}+N^{i} d t\right)\left(d x^{j}+N^{j} d t\right) .
$$

For the coordinates of the sections of constant time, we will employ the notation $\left\{x^{i}\right\} \equiv\left\{x^{1}, x^{2}, u\right\}$. In addition, we will assume that the coordinates $t$ and $u$ run over the real line, and impose that $\partial_{x^{a}}(a=1,2)$ are the two spacelike Killing vector fields. As a consequence, the metric must be independent of the coordinates $x^{a}$. This implies that the area $\mathcal{S}=\int d x^{1} d x^{2}$ appears as a constant global factor in the gravitational Hilbert-Einstein action and in the symplectic form for geometrodynamics. We can always absorb this area in Newton's constant. In order to do this, renormalization is required when the area $\mathcal{S}$ is infinite [20] (this case can be considered as the limit $\mathcal{S} \rightarrow \infty$ of the compact case). For convenience, we further set the effective Newton's constant obtained in this way equal to $1 / 8 \pi$.

The fundamental Poisson brackets and momenta canonically conjugate to $h_{i j}$ are then given by

$$
\begin{aligned}
& \left\{h_{i j}(u), \Pi^{k l}(\bar{u})\right\}=\delta_{i}^{(k} \delta_{j}^{l)} \delta(u-\bar{u}), \\
& \Pi^{i j}=\frac{1}{2} h^{1 / 2}\left(h^{i k} h^{j l}-h^{i j} h^{k l}\right) K_{k l},
\end{aligned}
$$

where $\delta_{j}^{i}$ is the Kronecker delta, $\delta(u)$ is the Dirac delta on the real line, the indices in parenthesis are symmetrized, and $h^{i j}$ is the inverse of the induced metric. Thus, the symplectic form on phase space is

$$
\Omega=\int d u \mathbf{d} \Pi^{i j} \wedge \mathbf{d} h_{i j}
$$

with the symbols $\mathbf{d}$ and $\wedge$ denoting the exterior derivative and product, respectively.

The gravitational system has two kinds of constraints: the Hamiltonian constraint $\mathcal{H}$ and the momentum constraints $\mathcal{H}_{i}$, which generate spatial diffeomorphisms. Instead of dealing with $\mathcal{H}$, we will consider its densitized version, $\tilde{\mathcal{H}} \equiv h^{1 / 2} \mathcal{H}$. In terms of phase-space variables, these constraints take the expressions

$$
\begin{aligned}
\tilde{\mathcal{H}} & \equiv-\frac{h}{2}{ }^{(3)} R+\left(h_{i k} h_{j l}+h_{i l} h_{j k}-h_{i j} h_{k l}\right) \Pi^{i j} \Pi^{k l}=0, \\
\mathcal{H}_{i} & \equiv-2 h_{i j} D_{k} \Pi^{k j}=0 .
\end{aligned}
$$

Here, $D_{i}$ is the covariant derivative compatible with the induced metric and ${ }^{(3)} R$ its curvature scalar. The time derivative of any function on phase space is then

$$
\dot{f}=\partial_{t} f+\left\{f, \int d u\left(\underset{\sim}{N} \tilde{\mathcal{H}}+N^{i} \mathcal{H}_{i}\right)\right\}
$$

where $\partial_{t}$ is the partial derivative with respect to the explicit time dependence and $\underset{\sim}{N}=h^{-1 / 2} N$ is the densitized lapse function.

Using that the metric does not depend on the coordinates $x^{a}$, it is not difficult to check that the momentum constraints $\mathcal{H}_{a}$ can be rewritten as

$$
\mathcal{H}_{a}=-2\left(h_{a i} \Pi^{i u}\right)^{\prime} .
$$

For the globally hyperbolic spacetimes with two commuting spacelike Killing vectors that we are studying, it is then possible to fix the gauge freedom associated with these constraints by demanding that, on the sections of constant time, the $u$-line is orthogonal to the group orbits spanned by $\partial_{x^{a}}$. This requirement can be rephrased in the form

$$
h_{a u}=0, \quad a=1,2 .
$$

To see that the above conditions lead to a well-posed gauge fixing, we must prove that, in general, the gauge orbits generated by the constraints $\mathcal{H}_{a}$ intersect transversely the surface that is defined on phase space by conditions (3.8) and the constraints of the system. This is equivalent to prove that the gauge-fixing conditions have non-vanishing Poisson brackets with $\mathcal{H}_{a}$. A straightforward calculation shows

$$
\left\{h_{a u}, \int d u N^{b} \mathcal{H}_{b}\right\}=h_{a b}\left(N^{b}\right)^{\prime} .
$$

Taking into account that $h_{a b}$ is positive definite, we conclude that these brackets are generally different from zero (it suffices to take $\left(N^{a}\right)^{\prime} \neq 0$ ).

We must also check that our gauge fixing is compatible with dynamics, in the sense that there exists a choice for the components $N^{a}$ of the shift vector (which are the Lagrange multipliers of $\mathcal{H}_{a}$ ) so that, on the constraint surface, the gauge conditions (3.8) are preserved by the dynamical evolution. In other words, modulo constraints and gauge-fixing conditions, $\dot{h}_{a u}$ must vanish for a particular choice of $N^{a}$. Recalling the independence of the metric on $x^{a}$, one can see that

$$
\dot{h}_{a u}=h_{a b}\left(N^{b}\right)^{\prime}+\underset{\sim}{4} h_{a b} h_{u u} \Pi^{b u},
$$

where use of the gauge conditions has been made after calculating Poisson brackets. On the other hand, the solution to $\mathcal{H}_{a}=0$ is given by 


$$
\Pi^{a u}=h^{a b} f_{b}(t)
$$

with $f_{a}$ being two arbitrary functions of $t$ that must be real, because $\Pi^{a u} \in \mathbb{R}$. Thus, our gauge fixing is in fact preserved by the evolution provided that

$$
\left(N^{a}\right)^{\prime}=-4 \underset{\sim}{N} h_{u u} h^{a b} f_{b}(t) .
$$

Let us now integrate both terms in the above equality over $u$, and contract the result with $f_{a}(t)$. If we assume that the components $N^{a}$ of the shift vanish in the limit of large $u$ 's, we get

$$
\int_{\mathbb{R}} d u \underset{\sim}{N} h_{u u} h^{a b} f_{a} f_{b}=0 .
$$

It is worth commenting that, together with the conditions $h_{a u}=0$, the requirement $\lim _{u \rightarrow \pm \infty} N^{a}=0$ can be viewed as ensuring that the group orbits spanned by $\partial_{x^{a}}$ are asymptotically orthogonal to the surfaces with coordinates $t$ and $u$. This requirement should hence be satisfied at least in spacetimes where those surfaces are everywhere orthogonal. Returning to Eq. (3.13), we note that, since the integrand in that expression is real, it has to vanish at least at one point if the integral is equal to zero. Taking into account that the two-metric $N h_{u u} h_{a b}$ is positive definite, it follows that $f_{a}=0$ at that point. The functions $f_{a}$ must then be identically zero, because they are independent of $u$. Therefore, one obtains that, when $N^{a}$ vanishes for large absolute values of $u$, the solutions to the constraints $\mathcal{H}_{a}=0$ and consistency conditions $\dot{h}_{a u}=0$ are

$$
\Pi^{a u}=0, \quad N^{a}=0
$$

The vanishing of $h_{a u}$ and $N^{a}$ implies that the spacetime metric is block-diagonal, so that the assumption $\lim _{u \rightarrow \pm \infty} N^{a}=0$ employed in our gauge fixing turns out to guarantee that the surface with coordinates $x^{a}$ is actually orthogonal to that described by $t$ and $u$.

After this partial gauge fixing, the canonically conjugate pairs $\left(h_{a u}, \Pi^{a u}\right)$ and the momentum constraints $\mathcal{H}_{a}$ are eliminated from the system. The remaining geometrodynamic momenta and components of the induced metric provide a canonical set of variables on the phase space of the gauge-fixed model. The line element of this reduced model has the form

$$
d s^{2}=-N^{2} d t^{2}+h_{u u}\left(d u+N^{u} d t\right)^{2}+h_{a b} d x^{a} d x^{b},
$$

where the metric functions depend only on the coordinates $t$ and $u$.

At this stage, it is convenient to carry out a change of metric variables from $h_{u u}$ and $h_{a b}$ to a set of functions $\left\{q^{\alpha}\right\} \equiv\{v, w, y, z\}$ that is analogous to that employed to describe the family of metrics (2.9). In this way, our discussion will be straightforwardly applicable to the analysis of pure gravitational plane waves. The change is such that the line element adopts the expression

$$
\begin{array}{r}
d s^{2}=e^{2 w-z+y / 2}\left[-e^{2 z} \underset{\sim}{N^{2}} d t^{2}+\left(d u+N^{u} d t\right)^{2}\right]+ \\
e^{z-y / 2}\left[\left(d x^{1}\right)^{2}-2 v d x^{1} d x^{2}+\left(v^{2}+e^{y}\right)\left(d x^{2}\right)^{2}\right] .
\end{array}
$$

Here, all metric functions are allowed to depend on the coordinates $u$ and $t$, and we have densitized the lapse using that

$$
h=e^{2 w+z+y / 2} .
$$

Similarly to the situation found for plane waves, the condition that the induced metric is positive definite is now easily imposed: it suffices to demand that the functions $q^{\alpha}$ are real. On the other hand, since the change of variables performed is just a point transformation, it is possible to find a set of momenta $\left\{p_{\alpha}\right\}$ that are canonically conjugate to our new metric variables. These momenta are

$$
p_{\alpha}=\Pi^{u u} \frac{\partial h_{u u}}{\partial q^{\alpha}}+\Pi^{a b} \frac{\partial h_{a b}}{\partial q^{\alpha}},
$$

where $h_{u u}$ and $h_{a b}$ are regarded as functions of $q^{\alpha}$. Using relation (3.3), we then obtain that, in terms of the extrinsic curvature and our metric variables,

$$
\begin{aligned}
& p_{v}=-h^{1 / 2} e^{-z-y / 2}\left(v K_{11}+K_{12}\right), \\
& p_{w}=-h^{1 / 2} e^{-z-y / 2}\left[\left(v^{2}+e^{y}\right) K_{11}+2 v K_{12}+K_{22}\right] \\
& p_{y}=-\frac{1}{2} h^{1 / 2} e^{-z+y / 2} K_{11} \\
& p_{z}=-h^{1 / 2} e^{-2 w+z-y / 2} K_{u u} .
\end{aligned}
$$

Finally, the gravitational model considered is still subject to two constraints, which are given by the restriction of $\mathcal{H}_{u}$ and $\tilde{\mathcal{H}}$ to the case in which the metric is independent of the coordinates $x^{a}$ and $h_{a u}=\Pi^{a u}=0$. After some trivial calculations, one can show that the momentum constraint $\mathcal{H}_{u}$ takes the form

$$
\mathcal{H}_{u}=-p_{w}^{\prime}+p_{w} w^{\prime}+p_{v} v^{\prime}+p_{y} y^{\prime}+p_{z} z^{\prime},
$$

and that the densitized Hamiltonian constraint has the expression

$$
\begin{aligned}
\tilde{\mathcal{H}}= & \frac{e^{2 z}}{16}\left[\left(y^{\prime}\right)^{2}+4\left(v^{\prime}\right)^{2} e^{-y}-4 z^{\prime}\left(4 w^{\prime}+y^{\prime}-5 z^{\prime}\right)+16 z^{\prime \prime}\right] \\
& +p_{v}^{2} e^{y}-2 p_{w} p_{y}-p_{w} p_{z}+4 p_{y}^{2} .
\end{aligned}
$$

\section{GAUGE FIXING AND SYMMETRY REDUCTION}

We will now apply the analysis of the previous section to the study of pure gravitational plane waves. We will complete the gauge fixing of the system and introduce symmetry conditions that ensure that all classical solutions are plane waves in vacuum, so that the reduced model that is obtained really describes the family of metrics (2.9). 
Let us first remove the gauge freedom associated with the constraint $\mathcal{H}_{u}$, which generates diffeomorphisms in the coordinate $u$. A way to fix this gauge is to provide a one-to-one correspondence between the coordinate $u$ and a metric variable. This can be done in the case of the plane wave metrics that we are considering, because the variable $z$ can then be chosen as a strictly increasing function of $u \in R$, namely, the function $z_{0}$ defined in Eq. (2.7). In order to fix the gauge freedom, we then introduce the condition

$$
\chi_{u} \equiv z-z_{0}=z+e^{-u}=0 .
$$

Since $z_{0}^{\prime} \neq 0$, the constraint $\mathcal{H}_{u}=0$ can be easily solved to find the momentum canonically conjugate to $z$ :

$$
p_{z}=p_{z}^{0} \equiv \frac{1}{z_{0}^{\prime}}\left(p_{w}^{\prime}-p_{w} w^{\prime}-p_{v} v^{\prime}-p_{y} y^{\prime}\right) .
$$

Therefore, if our gauge fixing is admissible, we can eliminate the canonical pair $\left(z, p_{z}\right)$ from the system.

Actually, condition (4.1) leads to a well-posed gauge fixing, because (modulo that condition)

$$
\left\{\chi_{u}, \int d u N^{u} \mathcal{H}_{u}\right\}=z_{0}^{\prime} N^{u} .
$$

Obviously, this Poisson bracket differs from zero when $N^{u} \neq 0$, because $z_{0}^{\prime}$ does not vanish at any point. On the other hand, if our gauge condition is compatible with the dynamical evolution, there must exist a choice for $N^{u}$ (i.e., the Lagrange multiplier of $\mathcal{H}_{u}$ ) such that

$$
\begin{aligned}
\dot{\chi}_{u} & =\left\{\chi_{u}, \int d u\left(\underset{\sim}{N} \tilde{\mathcal{H}}+N^{u} \mathcal{H}_{u}\right)\right\} \\
& =-p_{w} \underset{\sim}{N}+z_{0}^{\prime} N^{u}=0 .
\end{aligned}
$$

In the second line of this equation, we have substituted $z=z_{0}$. The condition $\chi_{u}=0$ is thus preserved by the evolution if

$$
N^{u}=\frac{p_{w} \underset{\sim}{N}}{z_{0}^{\prime}} .
$$

Note that the component $N^{u}$ of the shift given by this formula is always well-defined, because $z_{0}$ never vanishes. We hence conclude that the gauge fixing introduced is acceptable. It is worth remarking that, to arrive at this result, the only property of the function $z_{0}$ that has been used is that its derivative with respect to $u$ is everywhere finite and different from zero. In this sense, the explicit form of this function, as well as its range, are irrelevant.

After this gauge fixing, we obtain a reduced model whose phase space is described by the canonical set of variables $\left\{v, w, y, p_{v}, p_{w}, p_{y}\right\}$. The symplectic form is

$$
\Omega=\int d u\left(\mathbf{d} p_{v} \wedge \mathbf{d} v+\mathbf{d} p_{w} \wedge \mathbf{d} w+\mathbf{d} p_{y} \wedge \mathbf{d} y\right),
$$

and the system is subject to the densitized Hamiltonian constraint

$$
\tilde{\mathcal{H}}_{r} \equiv \tilde{\mathcal{H}}\left(z=z_{0}, p_{z}=p_{z}^{0}\right)=0 .
$$

In order to complete the reduction and attain a midisuperspace model for pure gravitational plane waves, we still need to introduce two types of conditions. Firstly, we have to impose that all classical solutions of the system are independent of the time coordinate, so that these solutions correspond to the family of plane wave metrics (2.9). Secondly, we have to find a condition that results in removing the gauge freedom associated with the densitized Hamiltonian constraint. Both tasks can be achieved in the following way.

We have shown at the end of Sec. II that, for metrics of the form (3.16) with $z=z_{0}$ and $N^{u}=-e^{z_{0}} \underset{\sim}{N}$, the time independence of the metric functions $v$ and $y$ on classical solutions is ensured by relations (2.10). Using Eqs. (3.19), those relations can be expressed as the conditions

$$
\begin{aligned}
& \chi_{1} \equiv p_{y}-\frac{e^{z_{0}}}{8}\left(y^{\prime}-2 z_{0}^{\prime}\right)=0, \\
& \chi_{2} \equiv 2 e^{y-z_{0}} p_{v}-v^{\prime}=0 .
\end{aligned}
$$

Taking into account identity (4.5), we see that these conditions guarantee that $v$ and $y$ do not depend classically on time provided that

$$
\chi_{0} \equiv p_{w}+z_{0}^{\prime} e^{z_{0}}=0 .
$$

When this is the case, it is clear that conditions (4.8) will also imply the time independence of the momenta $p_{v}$ and $p_{y}$. In addition, the momentum $p_{w}$ will not depend on time if the requirement $\chi_{0}=0$ is satisfied. The only phase-space variable that might then be time dependent on classical solutions is $w$. This possible time dependence can nevertheless be eliminated by demanding that

$$
\chi_{3} \equiv w-w_{0}=0,
$$

where $w_{0}$ is the function defined in Eq. (2.8). In this way, the function $w$ is fixed to take the same expression in terms of $v$ and $y$ as it adopts in pure gravitational plane waves. On the other hand, the constraint of the model can be rewritten after some manipulations as

$$
\begin{aligned}
\tilde{\mathcal{H}}_{r}= & \left(2 y^{\prime} \chi_{1}+e^{z_{0}-y} v^{\prime} \chi_{2}+2 w^{\prime} \chi_{0}-2 \chi_{0}^{\prime}\right) \frac{\chi_{0}}{2 z_{0}^{\prime}}+4 \chi_{1}^{2} \\
& +\frac{e^{2 z_{0}-y}}{4} \chi_{2}^{2}-2\left(\chi_{1}+e^{z_{0}} \chi_{3}^{\prime}\right) \chi_{0}+e^{z_{0}} \chi_{0}^{\prime} .
\end{aligned}
$$

So, once the symmetry conditions $\chi_{1}=\chi_{2}=0$ are imposed, the requirement $\chi_{0}=0$ is just a solution to $\tilde{\mathcal{H}}_{r}=0$. Therefore, the surface defined on phase space by $\chi_{I}=0(I=0, \ldots, 3)$ is simply a section of the constraint surface. In the following, we will show that the reduction to this section is consistent and that, in the reduction process, the condition $\chi_{3}=0$ allows us to fix the gauge freedom associated with $\tilde{\mathcal{H}}_{r}$. We will employ the symbol $\approx$ for weak identities, namely, identities that are satisfied modulo $\chi_{I}=0$. Besides, we will denote the set $\left\{\tilde{\mathcal{H}}_{r}, \chi_{1}, \chi_{2}, \chi_{3}\right\}$ by $\left\{\chi_{\mathcal{A}}\right\}$, with $\mathcal{A}=0, \ldots, 3$. 
To prove that the reduction is admissible, we first have to show that the conditions and constraint $\chi_{\mathcal{A}}=0$ constitute a second-class system on the section of the constraint surface that we are analyzing. Let us define

$$
\chi(g)=\int d u \sum_{\mathcal{A}} g_{\mathcal{A}} \chi_{\mathcal{A}},
$$

where $\left\{g_{\mathcal{A}}\right\}$ is any set of $C_{0}^{\infty}$ functions of the coordinate $u$, i.e., functions of $u$ that are infinitely differentiable and have compact support. Clearly, imposing $\chi_{\mathcal{A}}=0$ is equivalent to demand that $\chi(g)$ vanishes for all choices of the functions $g_{\mathcal{A}}$. The set $\left\{\chi_{\mathcal{A}}\right\}$ is then second-class provided that no combination of the form $\chi(g)$ (other than the zero constant) commutes weakly under Poisson brackets with all of the $\chi_{\mathcal{A}}$ 's. Using expression (4.11) for the constraint, and taking into account that all terms in that expression are quadratic in $\chi_{I}$ except the last one, we obtain

$$
\left\{\tilde{\mathcal{H}}_{r}, \chi(g)\right\} \approx-e^{z_{0}} g_{3}^{\prime} .
$$

Since $e^{z_{0}} \neq 0$ and the function $g_{3}$ is $C_{0}^{\infty}$, it follows that $\chi(g)$ commutes weakly with $\tilde{\mathcal{H}}_{r}$ if and only if $g_{3}$ vanishes. Let now $\bar{\chi}(g)$ be $\chi(g)$ for $g_{3}=0$. After some calculations, one gets

$$
\sum_{\mathcal{A}=1}^{2} g_{\mathcal{A}}\left\{\chi_{\mathcal{A}}, \bar{\chi}(g)\right\} \approx-\frac{1}{8}\left(e^{z_{0}} g_{1}^{2}+16 e^{y-z_{0}} g_{2}^{2}\right)^{\prime} .
$$

Given that the term in parenthesis is a sum of nonnegative functions and that $g_{1}, g_{2} \in C_{0}^{\infty}$, the vanishing of the Poisson brackets of $\bar{\chi}(g)$ with $\chi_{1}$ and $\chi_{2}$ turns out to imply that $g_{1}$ and $g_{2}$ must be the zero function. On the other hand, it is not difficult to check that

$$
\left\{\chi_{3}, \hat{\chi}(g)\right\} \approx-\left(e^{z_{0}} g_{0}\right)^{\prime},
$$

where $\hat{\chi}(g)$ denotes the restriction of $\chi(g)$ to the case in which $g_{0}$ is the only function in $\left\{g_{\mathcal{A}}\right\}$ that differs from zero. Recalling that $g_{0} \in C_{0}^{\infty}$, we see that the above Poisson bracket vanishes weakly provided that $g_{0}=0$. Therefore, $\chi(g)$ must be exactly equal to zero in order to commute weakly with the set $\left\{\chi_{\mathcal{A}}\right\}$, as we wanted to prove.

Let us now show that the section of the constraint surface determined by $\chi_{I}=0(I=0, \ldots, 3)$ is preserved by the dynamical evolution for a suitable choice of the densitized lapse. From expression (4.11) and the fact that the variables $\left\{\rho^{\alpha}\right\} \equiv\left\{v, y, p_{v}, p_{w}, p_{y}\right\}$ commute with $\chi_{0}$, we get

$$
\dot{\rho}^{\alpha}=\left\{\rho^{\alpha}, \int d u \underset{\sim}{N} \tilde{\mathcal{H}}_{r}\right\} \approx 0 .
$$

As a consequence, the variables $\rho^{\alpha}$ turn out to be time independent on classical solutions. To deduce this result, one actually needs not demand that $\chi_{3}$ vanishes. Moreover, one can then straightforwardly see that the symmetry conditions $\chi_{1}=\chi_{2}=0$ are preserved in time, and that, when these conditions are imposed, the solution to the constraint given by $\chi_{0}=0$ is invariant under the evolution, regardless of the value taken by the densitized lapse. On the other hand, employing again the expression for the constraint and that $\left\{\chi_{3}, \chi_{0}\right\}=\left\{w, \chi_{0}\right\}$, we obtain

$$
\dot{\chi}_{3} \approx-\left(e^{z_{0}} \underset{\sim}{N}\right)^{\prime} \approx \dot{w} .
$$

So, compatibility of the condition $\chi_{3}=0$ with the dynamical evolution ensures that the variable $w$ does not depend classically on time, and implies

$$
\underset{\sim}{N}=F(t) e^{-z_{0}} .
$$

The arbitrary function $F$ that appears in this expression can in fact be absorbed by means of the time redefinition $\tau=\int^{t} F(\bar{t}) d \bar{t}$. In this way, we arrive at $N=e^{-z_{0}}$. From Eqs. (4.5) and (4.9), the only non-vanishing component of the shift vector is then given by $N^{u}=-1$. We hence see that the reduction proposed for the system is acceptable and that the condition $\chi_{3}=0$ allows us to fix the value of the densitized lapse function. Therefore, this condition removes the gauge freedom associated with the constraint $\tilde{\mathcal{H}}_{r}$. In addition, we note that, in agreement with our previous discussion, all phase-space variables are indeed independent of the time coordinate on classical solutions after reducing the system to the section of the constraint surface defined by $\chi_{I}=0$.

In this reduction, the pair $\left(w, p_{w}\right)$ is eliminated via the gauge-fixing condition $\chi_{3}=0$ and the solution to the constraint $\chi_{0}=0$, while the symmetry conditions $\chi_{1}=\chi_{2}=0$ determine the momenta $p_{v}$ and $p_{y}$ in terms of the variables $v$ and $y$. The reduced model that is attained is free of constraints and possesses only two degrees of freedom, which are described by the metric variables $v$ and $y$. The line element for this reduced model can be obtained from Eq. (3.16) by substituting $z=z_{0}$, $w=w_{0}$, and the values obtained with our gauge fixing for $N^{u}$ and the densitized lapse function. This line element coincides formally with that given in Eq. 2.9) for pure gravitational plane waves. The only difference is that the functions $v$ and $y$ of the coordinate $u$ may now depend also on time. However, this time dependence is ruled out on classical solutions, as we have shown above. The metric may depend on time only when non-classical trajectories are allowed, e.g., in a quantum theory. Besides, it is clear that the set of classical solutions for our reduced model is precisely the family of gravitational plane waves in vacuum considered in Sec. II. As a consequence, our model describes in fact that family of plane waves.

Obviously, the dynamics of this reduced model is trivial, because the variables $v$ and $y$ remain constant in time under the classical evolution. The reduced Hamiltonian, which generates the time evolution via Poisson brackets, must hence vanish. Of course, the same conclusion is reached by considering the reduced action for the system. This action can be obtained from the Hilbert-Einstein action in the following manner. We adopt the $(3+1)$ decomposition of the metric explained in Sec. III, impose that 
the metric is independent of $x^{a}(a=1,2)$, and absorb the area that corresponds to these coordinates in Newton's constant, setting the resulting effective constant equal to $1 / 8 \pi$. Since all gravitational constraints are eliminated in the reduction process, we can make them equal to zero in the Hamiltonian form of the action. The reduced action $S_{r}$ that we are seeking can then be computed from the Lagrangian density $\Pi^{i j} \dot{h}_{i j}$. Substituting $\Pi^{a u}=0$, relations (3.18), and the values taken in our model by the variables $\left(w, z, p_{v}, p_{w}, p_{y}, p_{z}\right)$, we get

$$
S_{r}=\int d t d u \frac{e^{z_{0}}}{8}\left(y^{\prime} \dot{y}+4 v^{\prime} e^{-y} \dot{v}\right) .
$$

In arriving at this result, we have disregarded surface terms that are evaluated on sections of constant time, for they do not modify the reduced Hamiltonian (nor affect the symplectic structure). We see that the reduced action is linear in time derivatives. This implies that the reduced model has indeed a vanishing Hamiltonian in the set of coordinates adopted.

Given that the dynamics is trivial, all relevant information for quantizing the system is encoded in its symplectic structure. The symplectic form $\Omega_{r}$ on the phase space of the reduced model is provided by the pull-back of the form 4.6 to the surface determined by the conditions $\chi_{I}=0(I=0, \ldots 3)$. Taking into account that $\chi_{0}=0$ ensures that $p_{w}$ is a fixed function of the coordinate $u$ (because so is $z_{0}$ ), we obtain

$$
\Omega_{r}=\int d u \frac{e^{z_{0}}}{8}\left[\mathbf{d} y^{\prime} \wedge \mathbf{d} y+4 \mathbf{d}\left(v^{\prime} e^{-y}\right) \wedge \mathbf{d} v\right]
$$

Actually, this form could have also been deduced from the reduced action (4.19). The quantization of the system can then be attained by quantizing the algebra of Poisson brackets that follows from this symplectic form. In principle, the algebra seems rather complicated. This situation contrasts with that found in other midisuperspace models which describe spacetimes with two commuting spacelike Killing vectors, like, e.g., the Gowdy model [2], where the Poisson algebra obtained after (an almost complete) gauge fixing is simple, although the complexity of the system shows up in the expression for the reduced Hamiltonian. Nonetheless, it turns out that there exists at least a class of pure gravitational plane waves for which this Poisson algebra becomes manageable, namely, the case of linearly polarized plane waves. From now on, we will restrict our discussion to this type of waves.

\section{LINEARLY POLARIZED PLANE WAVES}

Pure gravitational plane waves for which the metric function $v$ vanishes are called linearly polarized $[13 \|$. One can actually impose the vanishing of $v$ as an additional symmetry on the midisuperspace model for plane waves in source-free gravity that we have studied. This can be done, for instance, by including the condition $\chi_{4} \equiv v=0$ in the process of gauge fixing and symmetry reduction explained in the previous section. Similar arguments to those presented for general plane waves in vacuum gravity show that the set formed by $\chi_{4}$ and $\chi_{\mathcal{A}}$ (with $\mathcal{A}=0, \ldots, 3)$ is second-class on the section of the constraint surface defined by the requirements $\chi_{I}=\chi_{4}=0$ $(I=0, . .3)$. Here, we have employed the notation introduced in Sec. IV. On the other hand, we have already seen that the metric variable $v$ is invariant under the dynamical evolution when the conditions $\chi_{I}=0$ are satisfied, so that the symmetry condition $\chi_{4}=0$ is compatible with dynamics. Therefore, the reduction obtained by demanding that $\chi_{I}$ and $\chi_{4}$ vanish is fully consistent.

The reduced model that one obtains in this manner is such that its phase space can be described by a single metric variable: the field $y$. As it happens for plane waves with two polarizations, this variable depends on the coordinate $u$ and, although it might in principle evolve in time, its time dependence is forbidden on classical solutions. It is easy to check that the action $\bar{S}_{r}$ and the symplectic form $\bar{\Omega}_{r}$ for this reduced model coincide in fact with those given in Eqs. (4.19) and (4.20) when particularized to the case $v=0$. Defining

$$
Y=\frac{e^{z_{0} / 2} y}{2 \sqrt{2}}
$$

we can then write

$$
\bar{S}_{r}=\int d t d u Y^{\prime} \dot{Y}, \quad \bar{\Omega}_{r}=\int d u \mathbf{d} Y^{\prime} \wedge \mathbf{d} Y,
$$

where we have used that $z_{0}$ is a fixed function of $u$ and neglected surface contributions to the action that come from sections of constant time. Note that the reduced action $\bar{S}_{r}$ corresponds indeed to a system with zero Hamiltonian and symplectic structure given by $\bar{\Omega}_{r}$.

Like in the case of waves with two polarizations, the dynamics of the reduced model for linearly polarized waves is trivial in the set of coordinates adopted. The function $Y$ is constant in time and the reduced Hamiltonian vanishes. Hence, in order to quantize the system, we only need to quantize the algebra of Poisson brackets that follows from the symplectic form $\bar{\Omega}_{r}$.

On the other hand, employing that the coordinate $u$ runs over the whole real axis, we can express the function $Y$ as the Fourier transform

$$
Y=\frac{1}{\sqrt{2 \pi}} \int_{0}^{\infty} \frac{d k}{\sqrt{2 k}}\left(a_{k} e^{-i k u}+a_{k}^{\star} e^{i k u}\right) .
$$

The complex functions $a_{k}$ and $a_{k}^{\star}$, with $k \in \mathbb{R}^{+}$, provide then a complete set of variables on the phase space of the reduced model. These functions of $k$ might depend as well on time; however, since $Y$ is time independent on classical solutions, they turn out to be classical constants of motion. Substituting the above expression for $Y$ in the symplectic form, we obtain 


$$
\Omega_{r}=i \int_{0}^{\infty} d k \mathbf{d} a_{k}^{\star} \wedge \mathbf{d} a_{k} .
$$

As a consequence, the only non-vanishing Poisson brackets between the phase-space variables $a_{k}$ and $a_{k}^{\star}$ are

$$
\left\{a_{\bar{k}}, a_{k}^{\star}\right\}=-i \delta(\bar{k}-k) .
$$

In addition, the fact that the function $Y$ is real implies that $a_{k}^{\star}$ must be the complex conjugate of $a_{k}$. Therefore, we can interpret the algebra of Poisson brackets for our model as corresponding to an infinite set of harmonic oscillators, described by the annihilation and creation like variables $a_{k}$ and $a_{k}^{\star}$, respectively.

The quantization of this algebra can be performed by standard methods. For instance, one can simply introduce a Fock representation [25]. In that case, our phasespace variables are represented as annihilation and creation operators, $\hat{a}_{k}$ and $\hat{a}_{k}^{\star}$, and the Hilbert space of physical states can be constructed by the repeated action of the creation operators on a vacuum, which is destroyed by all the operators $\hat{a}_{k}$. The algebra of commutators is given by the quantum analog of Eq. (5.5),

$$
\left[\hat{a}_{\bar{k}}, \hat{a}_{k}^{\star}\right]=\delta(\bar{k}-k),
$$

where we have set $\hbar=1$, and $\bar{k}, k \in \mathbb{R}^{+}$. Assuming that the vacuum has unit norm, the inner product is totally determined by requiring that the relations under complex conjugation between the variables $a_{k}$ and $a_{k}^{\star}$ are realized in the quantum theory as adjointness relations between operators. In particular, it is then possible to find normalized states which are formally similar to the states with $n$ particles or to the coherent states of ordinary quantum field theory in flat spacetime.

In this way, one attains a consistent and well-defined mathematical framework for analizying the quantum physics of spacetimes with two commuting Killing vectors that describe pure gravitational plane waves with linear polarization.

\section{CONCLUSIONS AND FURTHER COMMENTS}

Pure gravitational plane waves have been considered as a special class of spacetimes with two commuting spacelike Killing vector fields. We have discussed the structure of the reduced phase space and the quantization of this family of solutions to vacuum general relativity.

We have first seen that, with a suitable choice of coordinates, the metric for these plane waves can be cast in a $3+1$ form in which the values of the lapse and shift are fixed and all metric functions depend just on a coordinate of the sections of constant time, namely, the coordinate $u \in \mathbb{R}$. The other two coordinates of these sections (which run over the real axis) correspond to Killing vectors, with group orbits that are orthogonal to the surface described by $u$ and the time coordinate. We have shown that the determinant of the metric for these group orbits can be chosen as a strictly increasing function of the coordinate $u$, with range given by the interval $(0,1)$. In addition, we have solved explicitly Einstein equations in vacuum, and introduced metric variables whose reality ensures that the induced metric is positive definite. The family of metrics that we have obtained in this manner represent the most general plane wave solution in source-free gravity, with the only caveat that they do not describe the whole spacetime which can in principle be covered with harmonic coordinates. These metrics are determined by two arbitrary functions of the coordinate $u$, i.e., the functions $v$ and $y$.

With the aim at studying this family of metrics, we have considered the Hamiltonian formalism for spacetimes that admit two commuting spacelike Killing vectors. Assuming the spatial topology to be that of $\mathbb{R}^{3}$, it has been proved that the condition that there exists a surface orthogonal to the group orbits removes the gauge freedom related with diffeomorphims of the coordinates which correspond to the Killing vectors. We have then particularized our analysis to the case of pure gravitational plane waves. Using that the determinant of the metric for the group orbits is an increasing function of the coordinate $u$, we have been able to eliminate the degrees of freedom associated with diffeomorphisms of the $u$-line. In order to complete the gauge fixing and guarantee that the classical solutions of the system are plane waves, we have next introduced two types of requirements. On the one hand, we have imposed the symmetry conditions (4.8), which can be interpreted as relations between the metric and the extrinsic curvature that are satisfied for plane waves. Once these conditions are included, the densitized Hamiltonian constraint, which is the only constraint that still remains on the system, is easily solved. Moreover, all metric variables are then classically time independent, except possibly the variable $w$, defined by means of Eq. (3.16). On the other hand, we have demanded that $w$ adopts the same expression in terms of $v$ and $y$ as it does in pure gravitational plane waves. We have shown that, in this way, the gauge freedom associated with the densitized Hamiltonian constraint is totally removed and the time dependence of $w$ is ruled out on classical solutions.

After this process of gauge fixing and symmetry reduction, we have arrived at a midisuperspace model which is free of constraints and whose line element coincides formally with that for plane waves in vacuum general relativity, although the two functions $v$ and $y$ that determine the metric may depend not just on the coordinate $u$, but also on time if non-classical trajectories are allowed. The phase space of this reduced model has only two degrees of freedom, which are described by the variables $v$ and $y$. These variables remain constant in time on all classical solutions, so that the dynamical evolution on phase space is given by the identity map. This implies that the reduced Hamiltonian vanishes in the set of coordinates employed. The same conclusion has been 
reached by computing the reduced Hilbert-Einstein action. Up to surface contributions on sections of constant time, this action is linear in time derivatives, so that it indeed corresponds to a system with vanishing Hamiltonian. Since the dynamics is trivial, all the information needed for quantizing the reduced model is provided by its symplectic structure. Starting with the symplectic form for spacetimes with two spacelike Killing vectors, and calculating the pull-back to the section of the constraint surface determined by the gauge-fixing and symmetry conditions imposed on our model, we have then obtained the symplectic form on the reduced phase space.

There exists at least a case in which the algebra of Poisson brackets that follows from this symplectic form is simple enough as to allow a straightforward quantization, namely, the case of linearly polarized plane waves. The restriction to this subfamily of plane waves in sourcefree gravity has been achieved by demanding that the function $v$ vanishes. This requirement can be viewed as an additional symmetry condition on the system. The phase space of the resulting reduced model is described by the metric variable $y$ or, equivalently, by the set of variables $\left\{a_{k}, a_{k}^{\star} ; k \in \mathbb{R}^{+}\right\}$. These variables are classical constants of motion obtained from the Fourier transform of the product of $y$ and a fixed function of the coordinate $u$. We have finally proved that the algebra of Poisson brackets for the reduced model of linearly polarized plane waves can actually be understood as corresponding to an infinite set of harmonic oscillators whose annihilation and creation like variables are $a_{k}$ and $a_{k}^{\star}$, respectively. The quantization of the model can then be readily performed by introducing a Fock representation for these variables.

The mathematical framework constructed in this manner can be used to study the quantum physics of pure gravitational plane waves with linear polarization. In particular, one can try to define operators for the metric components and analyze the quantum fluctuations of the geometry. Another appealing possibility is to discuss the role played in the quantum theory by coherent states and see whether these are peaked around classical solutions, comparing the results with those obtained for linearly polarized waves with cylindrical symmetry 3,12 . The consideration of these issues will be the subject of a future research.

Another direction for further investigation consists in introducing a scalar field in the model and studying its interaction with gravitational plane waves that are linearly polarized. This interaction has already been discussed in the general case of waves with two polarizations using a semiclassical approximation, i.e., considering only the quantization of the scalar field [24]. It would be interesting to investigate whether the conclusions reached with this semiclassical analysis are valid to some extent in a purely quantum theory.

Let us conclude with a couple of remarks about our reduced model for linearly polarized plane waves. The first comment refers to the algebra of Poisson brackets that we have found. It is not difficult to see that, at a given instant of time, the values of the metric variable $y$ at two different points do not generally commute under Poisson brackets. This implies that, in the quantum theory, there cannot exist states in which the variable $y$ takes a welldefined value at every point on a section of constant time, even though the points on this section are spacelike separated. In fact, this result is not so surprising. We have seen that the reduced Hamiltonian of the system vanishes in the set of coordinates adopted, so that the quantum evolution is dictated by the identity operator. Therefore, if it were possible to determine completely the value of $y$ on a section of constant time, the same would happen for the entire spacetime, including points with timelike separation. The second of our remarks concerns the vanishing of the reduced Hamiltonian. This conclusion is valid in the set of coordinates that we have employed to describe the spacetime. In another coordinate system, however, the Hamiltonian of our reduced model can generally differ from zero. Suppose, for instance, that we change coordinates from $u$ to $x=u-t$. Using the expression for the reduced action given by Eq. (5.2) in the linearly polarized case, one can prove that the reduced Hamiltonian of the system becomes then

$$
\int d x\left(\partial_{x} Y\right)^{2}=\int_{0}^{\infty} d k k a_{k} a_{k}^{\star}
$$

where $Y$ is the variable defined in Eq. (5.1), and we have substituted relation (5.3) after replacing the coordinate $u$ with $x$. In the new coordinate system, the interpretation of our reduced model as a collection of harmonic oscillators applies hence not only to the Poisson algebra, but also to the Hamiltonian that generates the time evolution.

\section{ACKNOWLEDGMENTS}

The authors are grateful to L. J. Garay and C. Anastopoulos for valuable discussions. They wish to thank also E. Verdaguer, who initially proposed the subject. G. A. M. M. acknowledges DGICYT for financial support under the Research Project No. PB94-0107. M. M. was supported by funds provided by a Basque Government FPI grant.

[1] A. Abramovici et al., Science 256, 325 (1992); First International LISA Symposium, Class. Quantum Grav. 14, 1397 (1997); P. R. Brady, T. Creighton, C. Cutler, and B. F. Schutz, Phys. Rev. D 57, 2101 (1998); K. S. Thorne, in Black Holes and Relativistic Stars, ed. R. M. Wald (University of Chicago Press, Chicago, in press), gr-qc/9706079, and references therein. 
[2] G. A. Mena Marugán, Phys. Rev. D 56, 908 (1997).

[3] A. Ashtekar and M. Pierri, J. Math. Phys. 37, 6250 (1996).

[4] K. Kuchař, Phys. Rev. D 4, 955 (1971).

[5] D. Korotkin and H. Samtleben, Phys. Rev. Lett. 80, 14 (1998).

[6] See, for instance, A. Ashtekar, R. S. Tate, and C. Uggla, Int. J. Mod. Phys. D 2, 15 (1993); H. Kastrup and T. Thiemann, Nucl. Phys. B399, 211 (1993); B425, 665 (1994); N. Manojlović and G. A. Mena Marugán, Phys. Rev. D 48, 3704 (1993); G. A. Mena Marugán, Phys. Rev. D 53, 3156 (1996).

[7] D. Korotkin and H. Nicolai, Nucl. Phys. B475, 397 (1996); J. Cruz, D. J. Navarro, and J. Navarro-Salas, hep-th/9712194 D. Korotkin and H. Samtleben, Class. Quantum Grav. 14, L151 (1997); Commun. Math. Phys. 190, 411 (1997); hep-th/9710210; J. Cruz, A. Miković, and J. Navarro-Salas, gr-qc/9802067.

[8] R. H. Gowdy, Ann. Phys. (N.Y.) 83, 203 (1974).

[9] B. K. Berger, Ann. Phys. (N.Y.) 83, 458 (1974); 156, 155 (1984).

[10] V. Husain and L. Smolin, Nucl. Phys. B327, 205 (1989).

[11] M. Allen, Class. Quantum Grav. 4, 149 (1987).

[12] A. Ashtekar, Phys. Rev. Lett. 77, 4864 (1996); R. Gambini and J. Pullin, Mod. Phys. Lett. A 12, 2407 (1997).

[13] D. Kramer, H. Stephani, M. MacCallum, and E. Herlt, Exact Solutions of Einstein's Field Equations (Cambridge University Press, Cambridge, England, 1980).

[14] E. Verdaguer, Phys. Rep. 229, 1 (1993), and references therein.

[15] J. Ehlers and W. Kundt, in Gravitation: an Introduction to Current Research, ed. L. Witten (Wiley, New York, 1962).

[16] D. R. Baldwin and G. B. Jeffrey, Proc. R. Soc. London A111, 95 (1926).

[17] H. Bondi, F. A. E. Pirani, and I. Robinson, Proc. R. Soc. London A251, 519 (1959).

[18] G. F. R. Ellis and M. A. H. MacCallum, Commun. Math. Phys. 12, 108 (1969); J. Wainwright and B. J. Marshman, Phys. Lett. 72A, 275 (1979); S. T. C. Siklos, in Relativistic Astrophysics and Cosmology, eds. X. Fustero and E. Verdaguer (World Scientific, Singapore, 1984).

[19] K. A. Khan and R. Penrose, Nature (London) 229, 185 (1971); P. Szekeres, J. Math. Phys. 13, 286 (1972); A. Feinstein and J. Ibáñez, Phys. Rev. D 39, 470 (1989); J. B. Griffiths, Colliding Waves in General Relativity (Clarendon Press, Oxford, 1991), and references therein; D. Tsoubelis and A. Wang, J. Math. Phys. 33, 1054 (1992).

[20] D. E. Neville, Class. Quantum Grav. 10, 2223 (1993).

[21] D. E. Neville, Phys. Rev. D 55, 766 (1997); 55, 2069 (1997); 56, 3485 (1997); 57, 986 (1998).

[22] R. Borissov, Phys. Rev. D 49, 923 (1994).

[23] R. M. Wald, General Relativity (University of Chicago Press, Chicago, 1984).

[24] G. W. Gibbons, Commun. Math. Phys. 45, 191 (1975); J. Garriga and E. Verdaguer, Phys. Rev. D 43, 391 (1991).

[25] See, for instance, J. Glimm and A. Jaffe, Quantum Physics. A Functional Integral Point of View, 2nd. ed. (Springer-Verlag, New York, 1987). 